\title{
Medición De Carga Mental De Trabajo En La Industria Automotriz En México
}

\author{
Miguel Macías Rivera, MA \\ Universidad Popular Autónoma del Estado de Puebla, México \\ Raúl Rocha Romero, PhD \\ Facultad de Estudios Superiores Zaragoza, UNAM, México
}

doi: 10.19044/esj.2016.v12n26p92 URL:http://dx.doi.org/10.19044/esj.2016.v12n26p92

\begin{abstract}
The primary objective of this research was to determine and show the relationship that exists between the mental workload perceived by personnel in the automotive assembly industry and the labor activity performed. The sample was constituted of fifty-six workers and the evaluation consisted of the application of the SCAM Test (Díaz, Hernández, Rolo, Galván, Fraile and Loayssa, 2009), in the employee's workplace, as well as observation of the activities performed and interviews with some of the workers. This instrument evaluates five dimensions of workload: cognitive demands and information complexity, health consequences for workers, task characteristics, temporal organization of work and work rate. Results show that professionals perceive higher levels of mental workload, in the dimensions of cognitive demands, task complexity, as well as characteristics of the task. In addition to these findings, technicians perceived higher levels in work rate and corresponding health consequences. There is also a relationship between demographic and labor characteristics with the mental load perceived in workers. Furthermore, the environmental characteristics at work were not a significant factor in the perception of mental load in this research.
\end{abstract}

Keywords: Mental workload, cognitive demands and labor health

\section{Resumen}

El objetivo principal de esta investigación fue determinar y mostrar la relación que existe entre la carga mental de trabajo percibida por empleados de la industria ensambladora automotriz y la actividad laboral desempeñada. La muestra estuvo constituida por cincuenta y seis trabajadores y la evaluación consistió de la aplicación de la prueba ESCAM (Díaz, Hernández, Rolo, Galván, Fraile y Loayssa, 2009), en sus lugares de trabajo, así como, observación de las actividades realizadas y entrevistas con algunos de los 
trabajadores. Este instrumento evalúa cinco dimensiones de carga mental: Demandas cognitivas y complejidad de la información, consecuencias para la salud, características de las tareas, organización temporal y ritmo de trabajo. Los resultados muestran que los profesionales perciben niveles mayores de carga mental en su trabajo, en las dimensiones de demandas cognitivas, complejidad de la tarea, así como características de la tarea. Además de esto, se encuentra que los técnicos perciben mayores niveles en ritmo de trabajo y consecuencias para la salud. Hay también una relación entre las características demográficas y laborales con la carga mental percibida en los trabajadores. Por otra parte, las características ambientales en el trabajo no fueron significativas en la percepción de carga mental en esta investigación.

Palabras Clave: Carga mental de trabajo, demandas cognitivas y salud laboral

\section{Introducción}

Es frecuente que las condiciones de trabajo deriven en fatiga mental que conlleva disminución del desempeño y reducción de la atención, lentitud de pensamiento y, en ocasiones, un aumento en el número de errores, olvidos y confusiones que a su vez llevan a un aumento de la probabilidad de que ocurran accidentes laborales (Hart y Wickens, 1990; Lauridsen y Tonnesen, 1990). Asimismo, no se deben olvidar las consecuencias negativas o nocivas para la salud del trabajador, como son, por ejemplo, la disminución de la motivación laboral, la inestabilidad emocional (irritabilidad, ansiedad, estados depresivos), baja autoestima, alteraciones somáticas y del sueño o aumento en el consumo de tabaco, drogas y alcohol (Sluiter, Croon, Meijman y FringsDresen, 2003).

El concepto carga mental de trabajo se ha relacionado con una serie de estructuras teóricas psicológicas, dándose un gran énfasis a su evaluación y cuantificación en diferentes áreas, principalmente en la industria de la aviación. Por otra parte, esta técnica de evaluación ha sido elaborada y probada en culturas distintas a la mexicana, como serían los casos de España, Brasil y Chile (Rolo, Díaz, Hernández, 2009; Ceballos, Paravic, Burgos y Barriga, 2014).

Los países en vías de desarrollo, como México, son objeto de un acelerado proceso de industrialización, encaminado a la maquila de productos con predominio de la industria automotriz. Este tipo de trabajo se caracteriza por tareas repetitivas, con altas demandas de atención, que implican una alta concentración por parte del trabajador y con poco control sobre las actividades realizadas, lo cual puede tener efectos negativos en la salud mental de los sujetos (Almirall, 2001; Cooper y Payne, 1980). 
Como ya se mencionó, se pretende encontrar la relación que existe entre la carga mental de trabajo y el cargo desempeñado por la persona en su ámbito laboral, con el objeto de analizar la situación a la que se enfrentan los trabajadores de la industria automotriz en México.

Establecer un modelo explicativo de la relación de carga mental de trabajo y el cargo desempeñado ayudará a determinar las condiciones de trabajo a las que se ven expuestos los trabajadores y, con ello, se podrán establecer lineamientos que permitan modificar el ambiente de trabajo en sus aspectos ergonómicos y organizacionales. Esto traerá beneficios tanto a los trabajadores, a los cuales les permitirá trabajar en un ambiente de salud y seguridad, como al sector empresarial y social. Al primero le permitirá obtener mayor productividad y calidad, al segundo porque se disminuirá el gasto debido a las ausencias de los trabajadores y el pago de incapacidades de los mismos, así como sanciones impuestas por los organismos de salud del estado.

\section{La industria automotriz en México}

La historia de la industria automotriz en México data de 1925 con la instalación de las líneas de ensamble de Ford, cuyo desarrollo en Estados Unidos se incrementaba notablemente; posteriormente, en 1935, llega la que a la postre se convertiría en el mayor fabricante de vehículos en el nivel mundial: General Motors, en tanto que en 1938 inicia operaciones Automex, que posteriormente se convertiría en Chrysler.

Todas ellas centraron su actividad operacional en el montaje de vehículos destinados al mercado local que anteriormente satisfacía su demanda con importaciones. Varias fueron las razones que movieron a los fabricantes norteamericanos en un inicio y posteriormente a los europeos y asiáticos a trasladar su centro de fabricación a México. Dombois (1990) señala las siguientes:

Reducción de los costos de producción. En virtud de que los costos de importación de juegos CKD (Completely Knocked Down) usados para el ensamble eran menores a los pagados por la importación de vehículos.

$>\quad$ Bajos costos de transporte.

$>\quad$ Bajos salarios. Principalmente en la mano de obra usada en tareas de montaje.

$>\quad$ Expectativas de un mercado factible de monopolizar.

La característica principal en todas las plantas automotrices era que se trabajaba con un nivel de productividad bajo, resultado de mínimas inversiones y falta de infraestructura. Es hasta después de la segunda guerra mundial cuando el gobierno orienta sus esfuerzos hacia la industrialización del país; ya para 1950 México da un giro en su estructura económica hasta ese entonces dependiente de la agricultura y adquiere un enfoque pleno hacia el 
desarrollo industrial. Situación que posteriormente fortaleció en gran medida al sector automotriz (Vicencio, 2007: 214).

En 2014 siete estados generaron el $\mathbf{8 4 . 2 \%}$ de la producción total de la fabricación de automóviles y camiones del país (Tabla 1), y dieron empleo al 75.3\% de personas ocupadas en esta actividad.

Tabla 1. Fabricación de automóviles y camiones por entidad federativa

Porcentajes del valor de la producción bruta 2014

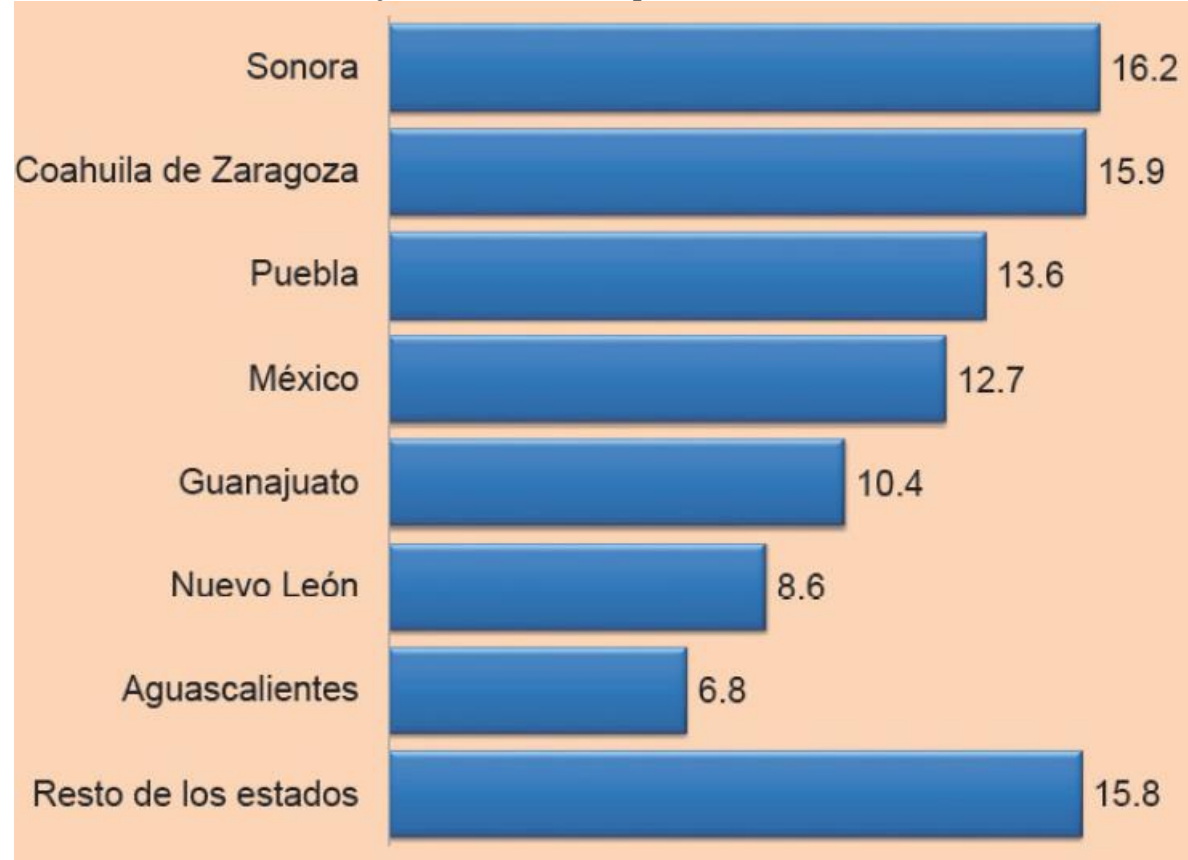

Fuente: AMIA con base de datos del INEGI.

Por lo que aporta la Industria automotriz a la producción manufacturera, se ubica entre las actividades más importantes después de la industria alimentaria (Tabla 2).

Tabla 2. Las industrias más importantes del sector manufacturero.

Porcentajes del PIB manufacturero a precios corrientes

\begin{tabular}{lrrrr}
\multicolumn{1}{c}{ Denominación } & 1993 & 2000 & 2005 & 2014 \\
\hline & 100.0 & 100.0 & 100.0 & 100.0 \\
Industria alimentaria & 24.1 & 20.2 & 22.7 & 23.6 \\
Industria automotriz & 11.2 & 13.5 & 12.1 & 16.9 \\
Industria química & 9.4 & 11.1 & 12.6 & 11.6 \\
Industrias metálicas básicas & 3.3 & 4.5 & 6.4 & 5.8 \\
Industria de las bebidas y del & 4.5 & 4.7 & 5.5 & 5.1 \\
tabaco & & & 5.5 \\
\hline
\end{tabular}

Fuente: AMIA con base de datos del INEGI. 
México, por el volumen de sus exportaciones de la industria automotriz, ocupó en 2014 el cuarto lugar a nivel mundial, después de Alemania, Japón y Estados Unidos de América. Las exportaciones de la Industria automotriz mexicana en 2014 representaron el $\mathbf{6 . 7 \%}$ del total de las exportaciones del mundo (Tabla 3 ).

Tabla 3. Principales países exportadores de la industria automotriz 2014

\begin{tabular}{clrc} 
Lugar & \multicolumn{1}{c}{ Países } & Millones de dólares & Porcentaje \\
\hline \hline & Exportaciones del mundo & 1266017 & 100.0 \\
1 & Alemania & 248911 & 19.7 \\
2 & Japón & 139048 & 11.0 \\
3 & Estados Unidos de América & 126139 & 10.0 \\
\hline 4 & México & 84258 & 6.7 \\
\hline 5 & Corea, República de & 72639 & 5.7 \\
6 & Canadá & 58377 & 4.6 \\
7 & España & 50838 & 4.0 \\
8 & Reino Unido & 50059 & 4.0 \\
9 & China & 48766 & 3.9 \\
10 & Francia & 44729 & 3.5 \\
11 & Bélgica & 43847 & 3.5 \\
12 & Italia & 33926 & 2.7 \\
\hline \hline
\end{tabular}

Fuente: AMIA con base de datos del International Trade Center

\section{El trabajo}

En sus orígenes, el trabajo fue en esencia la acción que el hombre realizaba para dominar o transformar la naturaleza con el propósito de obtener satisfactores para sus necesidades. La evolución de la sociedad ha conllevado el que estas necesidades sean cada día más complejas y alejadas de aquellas necesidades de orden predominantemente biológico de los remotos antepasados (Gutiérrez y Ángeles, 2012: 57).

El trabajo es cualquier actividad que produce un producto o un servicio para uso o de intercambio, y es importante por el papel que tiene como multiplicador de bienestar, ingreso, poder y prestigio. Es el proceso general a través del cual las sociedades producen bienes y servicios que satisfacen las necesidades humanas (Friedmann y Rosenman, 1974).

Según Rocha (2006), el trabajo es toda actividad que el hombre realiza para transformar su medio en los bienes que necesita para su subsistencia, lo que ha permitido su humanización. Pero no solo es fuente de riquezas materiales, sino también espirituales, porque forma parte muy importante del sentido que le asignan los individuos a sus propias vidas.

Como señalan Gutiérrez y Ángeles (2012: 58-59): “Cuando el trabajo es justamente remunerado en función de la percepción del esfuerzo realizado, permite que el ser humano obtenga dignidad, seguridad, satisfacción, tranquilidad y paz interior". 
Sin embargo, para Uribe (2014), el trabajo "digno" o trabajo "decente" es un principio, pero también es un deseo, que no siempre se ha alcanzado. Ha variado la distancia entre el deseo y los hechos; según el país, los gobernantes, los empresarios, los sindicatos y desde luego los trabajadores. La historia muestra que hoy en el mundo existen muchos tipos de relaciones de trabajo; en otras palabras, hay una diversidad de ambientes y climas económicos, sociales, políticos, organizacionales y laborales.

Debido a las características inherentes a la producción capitalista de mercancías, el trabajo se ha convertido en una actividad enajenada y enajenante, además de ser una fuente generadora de enfermedades y alteraciones psíquicas. La mayoría de los trabajadores están muy lejos de vivirlo como una actividad creativa que, a su vez les permita el desarrollo de sus potencialidades y facultades (Rocha, 2006: 56).

Pettigrew (1988) postula que es evidente que es mucho más fácil y ético modificar o cambiar los ambientes laborales que cambiar a los individuos. En este sentido, Schabracq, Cooper, Travers y Van Maanen (2001) evidencian que es más viable y más económico promover hábitos saludables de los trabajadores que asumir y pagar las consecuencias de no propiciar entornos saludables. Además, se sabe que un menor nivel de estrés y una mejor salud mental del trabajador están asociados con un mejor desempeño en el puesto.

\section{Carga mental de trabajo}

Las demandas cognitivas exigidas en el desempeño de un amplio número de trabajos hacen que el concepto carga mental adquiera una importancia significativa para el área de la prevención de riesgos laborales y, en concreto, para el bienestar físico y psicológico de los trabajadores (Rodríguez y Róldan, 2014).

Desde el punto de vista ergonómico, la causa principal de los errores durante el proceso productivo es el diseño inadecuado de las tareas y de los lugares de trabajo, lo que provoca una mayor cantidad de accidentes, incrementa la presencia de enfermedades y la duración de las incapacidades, asimismo, ocasiona decisiones equívocas y baja productividad (Jung y Jung, 2001).

Los individuos al realizar cualquier actividad laboral, se ven obligados a tolerar una carga impuesta por el trabajo, sea esta física o mental. La carga de trabajo física está constituida por los requerimientos biomecánicos, fisiológicos y calóricos que la tarea impone a los sujetos, mientras que la carga de trabajo mental son los requerimientos de procesamiento cognitivo que la tarea requiere para su adecuada realización (González, 2006). Para este mismo autor, la introducción de nuevas tecnologías en la industria, que aceleran el proceso productivo, exige del trabajador la realización de nuevas tareas, en las 
cuales la carga de trabajo mental desempeña un papel cada vez más trascendente. Esta carga es un elemento a considerar en el diseño e instrumentación de nuevos sistemas y procesos productivos, o en la mejora de los existentes.

Como ya se mencionó, aunque los sistemas automáticos requieren del trabajador un menor esfuerzo físico, incrementan el esfuerzo mental hasta el punto donde las capacidades humanas son el límite para el desempeño. Así pues, los nuevos sistemas redistribuyen, en vez de reducir, la carga de trabajo en el sistema (Moray, 1982; Tsang y Wilson, 1997). Por su parte, Galster (2004) concluyó que la automatización cambia la naturaleza de las demandas de trabajo y acarrea problemas que incluyen el desequilibrio de la carga mental de trabajo. Los niveles excesivamente altos de carga mental pueden acarrear errores y fallas en el sistema, aunque las cargas bajas pueden traer complacencia y errores.

De este modo, la carga mental de trabajo es la resultante de las exigencias de diversos factores del medio ambiente de trabajo (riesgos físicos, químicos y biológicos, factores tecnológicos y de seguridad, entre otros), y de las condiciones de trabajo (contenido y organización del trabajo, duración, sistemas de remuneración, etcétera), que están determinados por el proceso de trabajo vigente en la empresa (Neffa, 1988).

En función de sus manifestaciones somáticas, pueden ser agrupadas en sobrecarga cuantitativa y subcarga cualitativa mentales. La sobrecarga mental indica situaciones de tensión prolongada, como pueden ser niveles elevados de concentración o atención sostenida, supervisión estricta, conciencia de peligrosidad del trabajo, altos ritmos laborales, etcétera; por su parte, la subcarga mental se refiere a la imposibilidad de desarrollar y hacer uso de la capacidad psíquica, es decir, al hecho de realizar actividades sin contenido y sin significado para los trabajadores, debido a la pérdida del control sobre el trabajo al estar subordinados al movimiento de la máquina o a los instrumentos utilizados, a la descalificación del trabajo resultado de la separación entre su concepción y ejecución, así como por la parcelación del mismo que redunda en monotonía y repetitividad (Rocha, 2005).

La intensidad de la carga mental en el ambiente de trabajo está determinada por las características de la tarea que se está desempeñando. De acuerdo con la norma ISO 10075-2 (1996) algunos de los elementos que influyen en la intensidad son:

Ambigüedad de la tarea meta.

Complejidad de los requerimientos de la tarea.

Estrategias a seguir cuando existen múltiples requerimientos o cuando hay que desarrollar diversas tareas al mismo tiempo.

Adecuación y precisión de la información, que ésta no sea ambigua o que sea redundante. 
Discriminación de señales.

Compatibilidad de los controles con la tarea.

Procesamiento paralelo contra procesamiento serial.

Retraso en el tiempo de respuesta.

Carga en la memoria de trabajo y en la memoria de largo plazo.

Toma de decisiones

Requerimientos de coordinación de muchas dimensiones de conductas motoras: movimientos de traslación y rotación.

$>\quad$ Conducta de localización que requiere diferentes operaciones del operador.

$>\quad$ Consecuencias de errores en el desempeño humano y la tolerancia al error por parte del sistema.

$>\quad$ Diseño adecuado de las condiciones ambientales.

$>\quad$ Oportunidades para la interacción social al tomar decisiones ayudadas por el diseño de las tareas y del equipo.

$>\quad$ Dependencia de otros en el desempeño de tareas.

$>\quad$ Cambios en los requerimientos de la tarea.

$>\quad$ Presión de tiempo.

Los requerimientos temporales impuestos por la tarea son factores que incrementan la carga de trabajo mental, en función de la presencia de las siguientes características:

$>\quad$ Duración de las horas de trabajo.

$>\quad$ Tiempo de descanso entre días o turnos de trabajo sucesivos.

$>\quad$ El horario en el cual el sujeto trabaja.

$>\quad$ Trabajo por turnos.

$>\quad$ Pausas de descanso. Cambios en las tareas con diferentes demandas de trabajo o con grupos de carga mental de trabajo (ISO, 1996).

Se ha encontrado que la carga mental de trabajo tiene efectos en la salud física, por ejemplo, síntomas o quejas generales de salud, indicadores de enfermedad cardiaca coronaria y problemas músculo-esqueléticos. Existen reportes consistentes de asociación transeccional entre carga mental de trabajo percibida y quejas de salud (Repetti, 1993). En tres estudios de este tipo, que incluyen controles demográficos, se encontró relación entre las demandas del trabajo, las molestias físicas y psicosomáticas y problemas con el sueño, así como una variedad de síntomas tales como problemas digestivos, dolor de cabeza y mareos (González, 2006).

También se han realizado numerosas investigaciones enfocadas a la enfermedad cardiaca coronaria como un efecto asociado a la sobrecarga mental crónica de trabajo. Algunos incluyen evaluaciones de salud, como el realizado por Theorell y Floderus-Myrhed (1977) con 5000 trabajadores 
suecos de la construcción, cuyos resultados indican que los reportes de altos niveles de carga mental de trabajo percibida fueron asociados con un incremento del riesgo, ajustado por edad de infarto al miocardio.

Hitchen, Brodie y Arnés (1980) demostraron que la carga mental de trabajo tiene efectos sobre la variabilidad del ritmo cardiaco, cuando la carga aumenta, el ritmo disminuye y al mismo tiempo, la frecuencia respiratoria se incrementa. Charnock y Manenica (1978) encontraron que en aquellas tareas cuyo componente físico es poco significativo, tales como tareas mentales, tareas de tiempo de reacción y tareas ligeras repetitivas, se presentan efectos en el patrón del ritmo cardiaco.

Los efectos de la carga mental de trabajo en la presencia de desórdenes músculo-esqueléticos han sido demostrados en diversos estudios y principalmente se manifiestan en molestias en el cuello y en los hombros (Hanson, Schellekens, Veldman, y Mulder, 1993; Leino y Hanninen, 1995). Por su parte, Lundberg (1995) realizó un estudio entre trabajadores de línea de ensamble que a pesar de tener poca carga de trabajo física presentaban una alta incidencia de quejas de dolor de espalda. Sin embargo, las demandas mentales eran altas, sobre todo en las mujeres. Se comprobó la importancia de la carga mental de trabajo en el desarrollo de los síntomas músculo-esqueléticos.

La sobrecarga mental cuantitativa se ha asociado con tabaquismo y se relacionó significativamente a numerosos síntomas o indicadores de estrés, pobre motivación, baja autoestima, ausentismo y consumo de alcohol. Mientras que la sobrecarga mental cuantitativa está ligada a insatisfacción, depresión, irritación y síntomas psicosomáticos (González, 2006).

La subcarga mental cuantitativa también ha sido identificada como un estresor. El tener poco que hacer, la monotonía y el trabajo rutinario provocan aburrimiento y provoca falta de atención. El aburrimiento y la falta de cambios fueron predictores significativos de ansiedad, depresión y de insatisfacción en el trabajo, así como de baja autoestima (Hurrell, Murphy, Sauter y Cooper, 1988). La falta de estimulación puede ser particularmente dañina en la noche, cuando el individuo puede tener dificultad para ajustarse a los cambios en los patrones de sueño, pues no recibe estimulación en el trabajo para mantenerse despierto.

La sobrecarga y la subcarga mental cualitativa puede ser también una potente fuente de estrés psicológico y está asociada con las reacciones afectivas de los trabajadores hacia sus trabajos. La sobrecarga cualitativa ocurre cuando los individuos creen que no tienen las habilidades o capacidades para desarrollar satisfactoriamente sus tareas, y ha sido ligada a bajos niveles de autoestima (González, 2006). La subcarga mental cualitativa puede ser tan dañina como la sobrecarga, en la medida en que el individuo no tiene la oportunidad de usar las habilidades adquiridas o de desarrollar totalmente su potencial. Ambas se manifiestan en insatisfacción en el trabajo, pero 
diferencialmente, la subcarga mental cualitativa trae consigo pobre motivación, y alta rotación de personal, mientras que la sobrecarga mental cualitativa está asociada con tensión y baja autoestima (González, 2006).

Minowa (2000) realizó un estudio con ingenieros, encontrando que el $63.5 \%$ de los sujetos reportó carga mental de trabajo cualitativa, y el $36 \%$ sintió carga mental de trabajo cuantitativa. Al analizar cuántos de estos sujetos manifestaron padecer insomnio, el $25.5 \%$ de los sujetos con carga mental cualitativa lo padecen y el $11 \%$ de los que no manifestaron carga mental cualitativa. En forma similar, estados depresivos se encontraron en el $40.3 \%$ de los sujetos con carga mental cualitativa, y en $18.7 \%$ de los que no la tuvieron. Así, demostró que la carga mental cualitativa tiene mayores efectos en la salud mental que la carga mental cuantitativa.

La carga mental de trabajo ya sea cualitativa o cuantitativa puede tener efectos psicológicos y fisiológicos de corto plazo, acompañando la variabilidad diaria en el lugar de trabajo, siendo los efectos más importantes:

Humor y ansiedad. La evidencia señala que el incremento de la carga mental de trabajo puede tener un efecto de corto plazo en el humor. En tres estudios en los que se buscaba la asociación entre carga mental de trabajo percibida y humor en el mismo día, se encontró que los sujetos que tuvieron una alta carga mental de trabajo, presentaron mal humor al final de la jornada (Bolger, De Longis, Kessler y Schilling, 1989). En un estudio realizado con radio controladores de policías, se examinó el efecto de corto plazo de la carga mental de trabajo en la tensión y ansiedad. Hubo una asociación significativa entre la carga mental de trabajo percibida y la ansiedad al final del turno, pero no entre un indicador objetivo de carga mental de trabajo y humor (Kirmeyer y Dougherty, 1988).

$>\quad$ Activación. En un estudio de cambios de salud en controladores de tráfico aéreo (Rose, Jenkins y Hurts, 1978) se demostró que la presión sanguínea diastólica aumentó en días de alta carga de trabajo definida objetivamente. Este efecto fue mayor para los sujetos hipertensos. También hay evidencia de incremento en la presión sanguínea y ritmo cardiaco durante los periodos más demandantes del trabajo diario de paramédicos (Jamner, Shapiro, Goldstein y Hug, 1991; Téllez, 2001). Además, algunas investigaciones suecas sugieren que el excesivo tiempo extra está asociado con el aumento de la secreción de adrenalina y aumento del ritmo cardiaco durante el día, en el trabajo y por la tarde, en la casa (Lundberg y Palm, 1989).

$>\quad$ Respuestas conductuales de corto plazo. Conway, Vickers, Ward y Rahe (1981) encontraron que los empleados incurren en conductas más insanas que pueden incrementar su activación, tales como fumar y beber café en días en los que la carga de trabajo es percibida como alta. 


\section{Método}

\section{Participantes}

La muestra estuvo formada por 56 participantes, todos ellos trabajadores pertenecientes a una empresa automotriz del Estado de Puebla, México. La distribución por grupos fue la siguiente: técnicos $(58,9 \%)$, profesionales $(41,1 \%)$. La media de edad de todos los participantes fue de 43,84 años $(\mathrm{DT}=9,87)$. Del total de la muestra $46(82,1 \%)$ eran hombres y 10 $(17,9 \%)$ eran mujeres.

Respecto al contenido general de los puestos de trabajo, el personal técnico tenía como función principal el ensamble de piezas en líneas productivas, algunos de ellos tenían actividades administrativas básicas como lista de asistencia, estadística de fallas y manejo de personal. El personal profesional tenía como funciones principales el manejo de base de datos y proyectos, redacción de reportes, informes y tareas de atención a las áreas productivas, asistencia a juntas tanto internas como en otras áreas. Los puestos de mayor jerarquía fueron jefe de turno y gerente que adicionalmente tenían funciones de administración de presupuestos, asistencia periódica a juntas con directores, toma de decisiones de su personal a cargo.

Criterios de inclusión: Participaron en el estudio aquellos trabajadores que dieron su consentimiento y que tenían al menos un mes laborando en su cargo de trabajo.

\section{Instrumento}

Para evaluar la carga mental, se utilizó el instrumento Escala Subjetiva de Carga Mental de trabajo (ESCAM). Esta es una escala multidimensional de valoración de la carga mental de trabajo a partir de la percepción de los trabajadores (Rollo-González, Díaz-Cabrera y Hernández Fernaud, 2009). Presenta cinco dimensiones de carga mental:

Demandas cognitivas y complejidad de la tarea. Esfuerzo mental que supone el desempeño del puesto de trabajo (tales como memorización, concentración, evitación de errores, toma de decisiones, grado de complejidad de las tareas a realizar en el trabajo.

$>\quad$ Características de las tareas. Describe este factor un grupo de características de las tareas realizadas en el puesto de trabajo (tales como el número de interrupciones, demandas de concentración, la necesidad de simultanear tareas, la cantidad de dificultades que surgen al aplicar nuevos procedimientos o programas informáticos).

$>\quad$ Organización temporal. valoraciones sobre la adecuación del tiempo del que dispone el trabajador para realizar las tareas.

$>\quad$ Ritmo de trabajo. Posibilidad que tiene el trabajador de organizar su tiempo en el desempeño de sus tareas, así como la potencial gravedad de sus errores en los resultados globales del trabajo.

Consecuencias para la salud. Agotamiento que el desempeño del 
puesto de trabajo produce en el trabajador.

Cada reactivo fue evaluado en una escala tipo Likert de 5 puntos, en donde 1 es el valor mínimo y representa carga mental baja, y 5 es el valor máximo y representa carga mental alta.

\section{Procedimiento}

Una vez que se definieron los puestos y los grupos tanto de profesionales como técnicos interesados en colaborar, se explicó detalladamente a los responsables de cada uno de los trabajadores el objetivo de la investigación. Todos los participantes fueron informados de las características prácticas del estudio en el que iban a colaborar, haciendo especial hincapié en que se trataba de una participación totalmente voluntaria y anónima. Siguiendo el procedimiento de aplicación de la técnica ESCAM, se realiza la aplicación del instrumento en sus puestos de trabajo.

Posteriormente se procede al análisis estadístico utilizando el programada estadístico SPSS v.23 para tal fin. En primer lugar, se realiza la valoración de la carga mental del puesto de trabajo en cada una de las dimensiones, posteriormente se realiza la valoración de carga mental con las variables organizacionales, demográficas y ambientales en cada una de las dimensiones.

\section{Resultados}

Con la intención de observar la relación que existe entre las variables de estudio, se procedió a aplicar un análisis de varianza y medias aritméticas ( $\mathrm{t}$ de Student) entre las variables dependientes (técnicos y profesionales) y cada dimensión del instrumento, para así conocer el nivel de carga mental de trabajo existente de cada reactivo. Los resultados se presentan en las tablas 4 a la 7 (Solo se presentan los resultados que tuvieron significancia estadística).

Los resultados muestran que en las demandas cognitivas y complejidad de la tarea (Tabla 4) prevalece sobrecarga mental a excepción del indicador 5 en profesionales que perciben una carga mental normal. Así, en características de la tarea (Tabla 5) solo los profesionales en el reactivo 6 perciben una carga mental alta. En cuanto al ritmo de trabajo (Tabla 6) los técnicos muestran tendencia hacia carga mental alta sin que por el momento sea crítica ésta. Por último, en consecuencias para la salud (Tabla 7) tanto profesionales como técnicos muestra carga mental alta a excepción del indicador 17 que presenta una percepción normal de carga mental. 
Tabla 4. Dimensión: Demandas cognitivas y complejidad de la tarea

\begin{tabular}{|l|c|c|c|c|}
\hline \multicolumn{1}{|c|}{ Reactivo } & \multicolumn{2}{|c|}{ Media } & \multirow{2}{*}{$\mathrm{t}$} & \multirow{2}{*}{$\mathrm{p}$} \\
\cline { 2 - 3 } & $\mathrm{G} 1^{*}$ & $\mathrm{G} 2 *$ & & \\
\hline $\begin{array}{l}\text { 2. La cantidad de memorización de información y material que } \\
\text { requiere mi trabajo es: }\end{array}$ & 3,42 & 3,91 & $-2,342$ & 0,023 \\
\hline $\begin{array}{l}\text { 4. Habitualmente en mi puesto de trabajo el número de decisiones que } \\
\text { debo toma es: }\end{array}$ & 3,61 & 4,26 & $-3,329$ & 0,002 \\
\hline 5. El nivel de ambigüedad de las decisiones a tomar en mi trabajo es: & 3,33 & 2,78 & 2,247 & 0,032 \\
\hline $\begin{array}{l}\text { 8. El nivel de esfuerzo mental necesario para evitar los errores en mi } \\
\text { trabajo es: }\end{array}$ & 3,48 & 4,04 & $-2,655$ & 0,011 \\
\hline
\end{tabular}

${ }^{*} \mathrm{G} 1$ : Técnicos, ${ }^{*} \mathrm{G} 2$ : Profesionales.

Tabla 5. Dimensión: características de la tarea

\begin{tabular}{|l|c|c|c|c|}
\hline \multirow{2}{*}{ Reactivo } & \multicolumn{2}{|c|}{ Media } & \multirow{2}{*}{$t$} & \multirow{2}{*}{$\mathrm{p}$} \\
\cline { 2 - 4 } & $\mathrm{G} 1 *$ & $\mathrm{G} 2 *$ & & \\
\hline $\begin{array}{l}\text { 14. En mi trabajo, puedo cometer algún error sin que incida en forma } \\
\text { critica sobre los resultados del trabajo: }\end{array}$ & 3,18 & 2,48 & 2,146 & 0,036 \\
\hline
\end{tabular}

${ }^{*} \mathrm{G} 1:$ Técnicos, ${ }^{*} \mathrm{G} 2$ : Profesionales.

Tabla 6. Dimensión: Ritmo de trabajo

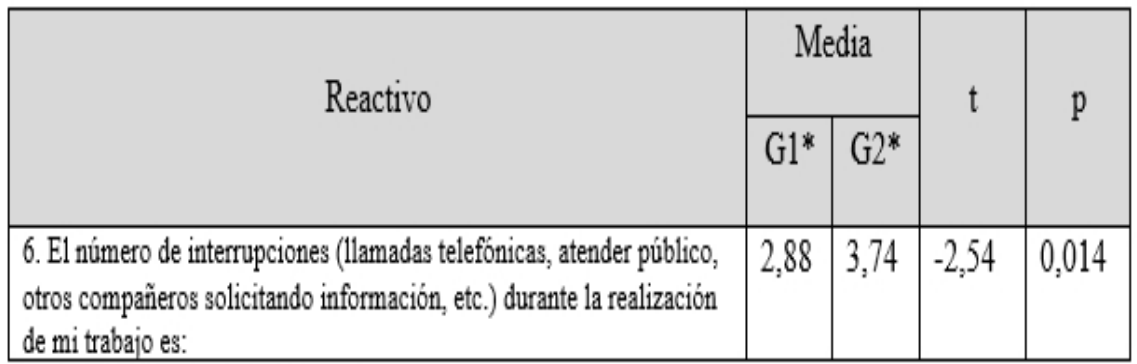

${ }^{*} \mathrm{G} 1$ : Técnicos, ${ }^{*} \mathrm{G} 2$ : Profesionales. 
Tabla 7. Dimensión: Consecuencias para la salud

\begin{tabular}{|c|c|c|c|c|}
\hline \multirow{2}{*}{ Reactivo } & \multicolumn{2}{|c|}{ Media } & \multirow{2}{*}{$\mathrm{t}$} & \multirow{2}{*}{$\mathrm{p}$} \\
\hline & G1* & $\mathrm{G} 2 *$ & & \\
\hline 9. E1 cansancio que me produce mi trabajo es: & 3,97 & 3,35 & 2,877 & 0,006 \\
\hline 15. A1 final de la jornada de trabajo me siento agotado: & 4,15 & 3,48 & 2,434 & 0,018 \\
\hline 17. Tengo dificultad para relajarme después del trabajo: & 3,03 & 2,48 & 1,886 & 0,065 \\
\hline
\end{tabular}

${ }^{*} \mathrm{G} 1$ : Técnicos, ${ }^{*} \mathrm{G} 2$ : Profesionales.

Para el caso de las variables demográficas, laborales y ambientales de trabajo, se procedió nuevamente a aplicar un análisis de varianzas y medias aritméticas (ANOVA) entre estas variables y cada dimensión del instrumento, para así conocer el grado de carga mental de trabajo existente de cada reactivo. Los resultados se presentan en las tablas 8 a la 14.

Los resultados muestran que los trabajadores de 15 a 24 años laborando en la empresa perciben la carga mental máxima producto del esfuerzo mental y concentración requerida, por otro lado, la carga mínima se encontró entre trabajadores que tienen menos de 5 años en la empresa (Tabla 8 ). En antigüedad en su puesto de trabajo los que perciben mayor carga mental fueron los de 21 a 25 años en un mismo puesto de trabajo, por otra parte, la carga mental mínima se muestra en trabajadores con menos de 1 año en su puesto (Tabla 9).

En cuanto a la rotación de turnos, los que presentan carga mental máxima fueron los trabajadores que tenían que rotar turnos y los que muestran la carga mental mínima fueron los del tercer turno (Tabla 10). En cuanto a la edad se encontró que los que tienen entre 40 a 49 años percibían la carga mental máxima sin que fuera muy alta, mientras que los que tenían menos de 30 años percibían la carga mental mínima (Tabla 11). En cuanto a la relación de carga mental y número de dependientes, los que presentaron mayor carga fueron los que tenían entre 1 a 3 dependientes, los que presentaron la menor carga mental fueron los que no tenían dependientes (Tabla 12). Con respecto a la escolaridad, los que presentaron mayor nivel de carga mental fueron los que tienen posgrado, así como los que presentan la menor carga mental fueron los que tienen un nivel de estudio de secundaria (Tabla 13). Por último, en lo referente a las horas de trabajo laboradas los que presentaban una carga mayor fueron los que trabajaban más de 10 horas al día, a su vez los que trabajaban entre 8 a 10 horas por día presentaban menor carga mental (Tabla 14).

En cuando a las características ambientales, no se encontró asociación de carga mental. 
Tabla 8. Dimensiones / antigüedad en la empresa

\begin{tabular}{|c|c|c|c|c|c|c|c|}
\hline \multirow{2}{*}{ Dimensión } & \multirow{2}{*}{ Reactivo } & \multicolumn{4}{|c|}{ Media } & \multirow{2}{*}{$F$} & \multirow{2}{*}{$\mathrm{p}$} \\
\hline & & $\mathrm{G1}^{*}$ & $\mathrm{G} 2 *$ & G3* & $\mathrm{G}^{*} *$ & & \\
\hline \multirow{2}{*}{$\begin{array}{l}\text { Demandas cognitivas } \\
\text { y complejidad de la } \\
\text { tarea. }\end{array}$} & $\begin{array}{l}\text { 3. El nivel de esfuerzo o } \\
\text { concentración mental que } \\
\text { requiere mi trabajo es: }\end{array}$ & 3,50 & 3,88 & 4,17 & 3,58 & 2,804 & 0,049 \\
\hline & $\begin{array}{l}\text { 5. El nivel de ambigüedad de } \\
\text { las decisiones a tomar en mi } \\
\text { trabajo es: }\end{array}$ & 4,0 & 3,29 & 2,67 & 3,26 & 3,206 & 0,031 \\
\hline \multirow{2}{*}{$\begin{array}{l}\text { Organización } \\
\text { temporal del trabajo. }\end{array}$} & $\begin{array}{l}\text { 18. El tiempo del que } \\
\text { dispongo para tomar las } \\
\text { decisiones exigidas por mi } \\
\text { trabajo es: }\end{array}$ & 3,50 & 3,82 & 2,89 & 3,11 & 4,793 & 0,005 \\
\hline & $\begin{array}{l}\text { 19. El tiempo de que } \\
\text { dispongo para realizar mi } \\
\text { trabajo es: }\end{array}$ & 4,0 & 3,71 & 3,0 & 3,26 & 2,965 & 0040 \\
\hline \multirow[b]{2}{*}{ Ritmo de trabajo. } & $\begin{array}{l}\text { 11. Es posible variar mi } \\
\text { ritmo de trabajo sin perturbar } \\
\text { el trabajo de mi seccion: }\end{array}$ & 2,0 & 3,75 & 2,94 & 3,26 & 3,778 & 0,016 \\
\hline & $\begin{array}{l}\text { 14. En mi trabajo, puedo } \\
\text { cometer algún error sin que } \\
\text { incida en forma crítica sobre } \\
\text { los resultados del trabajo: }\end{array}$ & 2,50 & 3,29 & 2,28 & 3,16 & 2,615 & 0,061 \\
\hline $\begin{array}{l}\text { Consecuencias para } \\
\text { la salud }\end{array}$ & $\begin{array}{l}\text { 16. Me siento agotado } \\
\text { cuando me levanto por la } \\
\text { mañana y tengo que } \\
\text { enfrentarme a otro dia de } \\
\text { trabajo: }\end{array}$ & 1,50 & 3,53 & 3,17 & 2,74 & 2,497 & 0,070 \\
\hline
\end{tabular}

|*G1: Menos de 5 años, ${ }^{*} \mathrm{G} 2: 5$-14 años, ${ }^{*} \mathrm{G} 3: 15-24$ años, ${ }^{*} \mathrm{G} 4: 25$ años o más.

Tabla 9. Dimensiones / antigüedad en el puesto

\begin{tabular}{|c|c|c|c|c|c|c|c|c|c|c|}
\hline \multirow{2}{*}{ Dimensión } & \multirow{2}{*}{ Reactivo } & \multicolumn{7}{|c|}{ Media } & \multirow{2}{*}{$\mathrm{F}$} & \multirow{2}{*}{$\mathrm{p}$} \\
\hline & & ${ }^{*} \mathrm{Gl}$ & ${ }^{\circ} \mathrm{G} 2$ & ${ }^{*} \mathrm{G} 3$ & "G4 & ${ }^{*} \mathrm{G} 5$ & ${ }^{\circ \mathrm{G} 6}$ & ${ }^{*} \mathrm{G} 7$ & & \\
\hline \multirow[t]{2}{*}{$\begin{array}{l}\text { Organización } \\
\text { temporal del } \\
\text { trabajo. }\end{array}$} & $\begin{array}{l}\text { 7. La cantidad de } \\
\text { dificultades que se } \\
\text { producen cuando se } \\
\text { introducen nuevos } \\
\text { procedimientos de } \\
\text { trabajo o programas } \\
\text { informáticos es: }\end{array}$ & 2,75 & 3,78 & 3,27 & 4,20 & 4,00 & 3,00 & 3,67 & 2,432 & 0,039 \\
\hline & $\begin{array}{l}\text { 13. En mi trabajo, tengo } \\
\text { que hacer más de una } \\
\text { tarea a la vez: }\end{array}$ & 1,75 & 4,26 & 3,91 & 3,80 & 4,00 & 4,50 & 3,67 & 5,081 & 0,000 \\
\hline
\end{tabular}

${ }^{*} \mathrm{G} 1$ : Menos de 1 año, ${ }^{*} \mathrm{G} 2: 1$ - 5 años, ${ }^{*} \mathrm{G} 3: 6-10$ años, ${ }^{*} \mathrm{G} 4: 11-15$ años, ${ }^{*} \mathrm{G} 5: 16-20$ años, ${ }^{*} \mathrm{G} 6: 21$ -25 años, ${ }^{*} \mathrm{G} 7: 26$ años o más. 
Tabla 10. Dimensiones / tipo de turno

\begin{tabular}{|c|c|c|c|c|c|c|c|}
\hline \multirow{2}{*}{ Dimensión } & \multirow{2}{*}{ Reactivo } & \multicolumn{4}{|c|}{ Media } & \multirow{2}{*}{$\mathrm{F}$} & \multirow{2}{*}{$\mathrm{p}$} \\
\hline & & $\mathrm{G}^{*}$ & $\mathrm{G} 2^{*}$ & $\mathrm{G}^{*} *$ & $\mathrm{G}^{*}{ }^{*}$ & & \\
\hline \multirow{3}{*}{$\begin{array}{l}\text { Caracteristicas de la } \\
\text { tarea. }\end{array}$} & $\begin{array}{l}\text { 6. E1 número de } \\
\text { interrupciones (llamadas } \\
\text { telefónicas, atender público, } \\
\text { otros compañeros solicitando } \\
\text { información, etc.) durante la } \\
\text { realización de mi trabajo es: }\end{array}$ & 3,56 & 2,00 & 1,00 & 3,22 & 3,398 & 0,024 \\
\hline & $\begin{array}{l}\text { 7. La cantidad de dificultades } \\
\text { que se producen cuando se } \\
\text { introducen nuevos } \\
\text { procedimientos de trabajo o } \\
\text { programas informáticos es: }\end{array}$ & 3,74 & 3,80 & 1,00 & 3,57 & 4,696 & 0,006 \\
\hline & $\begin{array}{l}\text { 13. En mi trabajo, tengo que } \\
\text { hacer más de una tarea a la } \\
\text { vez: }\end{array}$ & 3,96 & 3,40 & 1,00 & 4,13 & 3,889 & 0,014 \\
\hline
\end{tabular}

${ }^{*} \mathrm{G} 1$ : Mañana, ${ }^{*} \mathrm{G} 2:$ Tarde, ${ }^{*} \mathrm{G} 3$ : Noche, ${ }^{*} \mathrm{G} 4$ : Con rotación.

Tabla 11. Dimensiones / edad

\begin{tabular}{|c|c|c|c|c|c|c|c|c|}
\hline \multirow{2}{*}{ Dimensión } & \multicolumn{2}{|c|}{ Reactivo } & \multicolumn{3}{c|}{ Media } & \multirow{2}{*}{ F } & \multirow{2}{*}{ P } \\
\cline { 3 - 7 } & & ${ }^{*} \mathrm{G} 1$ & ${ }^{*} \mathrm{G} 2$ & ${ }^{*} \mathrm{G} 3$ & ${ }^{*} \mathrm{G} 4$ & ${ }^{*} \mathrm{G} 5$ & \\
\hline $\begin{array}{l}\text { Consecuencias para } \\
\text { la salud }\end{array}$ & $\begin{array}{l}\text { 17. Tengo dificultad para } \\
\text { relajarme después del } \\
\text { trabajo: }\end{array}$ & 1,5 & 2,86 & 3,22 & 2,67 & 2,50 & 2,380 & 0,064 \\
\hline
\end{tabular}

${ }^{*} \mathrm{G} 1$ : Menor de 30 años, ${ }^{*} \mathrm{G} 2: 30-39$ años, ${ }^{*} \mathrm{G} 3: 40-49$ años, ${ }^{*} \mathrm{G} 4: 50-59$ años, ${ }^{*} \mathrm{G} 5: 60$ años o más.

Tabla 12. Dimensiones / dependientes

\begin{tabular}{|c|c|c|c|c|c|c|}
\hline \multirow{2}{*}{ Dimensión } & \multirow{2}{*}{ Reactivo } & \multicolumn{3}{|c|}{ Media } & \multirow{2}{*}{ F } & \multirow{2}{*}{$\mathrm{p}$} \\
\hline & & $\mathrm{G}^{*}{ }^{8}$ & $\mathrm{G} 2 *$ & $\mathrm{G}^{*}$ & & \\
\hline $\begin{array}{l}\text { Consecuencias } \\
\text { para la salud }\end{array}$ & $\begin{array}{l}\text { 9. El cansancio que me } \\
\text { produce mi trabajo es: }\end{array}$ & 2,75 & 3,82 & 3,63 & 3,208 & 0,048 \\
\hline
\end{tabular}

${ }^{*} \mathrm{G} 1$ : Ninguno, ${ }^{*} \mathrm{G} 2: 1$ - $3,{ }^{*} \mathrm{G} 3: 4$ o más. 
Tabla 13. Dimensiones / nivel de estudios

\begin{tabular}{|c|c|c|c|c|c|c|c|c|}
\hline \multirow{2}{*}{ Dimensión } & \multirow{2}{*}{ Reactivo } & \multicolumn{5}{|c|}{ Media } & \multirow{2}{*}{$\mathrm{F}$} & \multirow{2}{*}{ p } \\
\hline & & ${ }^{*} \mathrm{G} 1$ & ${ }^{*} \mathrm{G} 2$ & ${ }^{*} \mathrm{G} 3$ & ${ }^{*} \mathrm{G} 4$ & ${ }^{*} \mathrm{G} 5$ & & \\
\hline \multirow{2}{*}{$\begin{array}{l}\text { Demandas } \\
\text { cognitivas y } \\
\text { complejidad de } \\
\text { la tarea. }\end{array}$} & $\begin{array}{l}\text { 4. Habitualmente en mi } \\
\text { puesto de trabajo el } \\
\text { número de decisiones que } \\
\text { debo toma es: }\end{array}$ & 3,63 & 3,57 & 3,71 & 4,25 & 4,80 & 4,060 & 0,006 \\
\hline & $\begin{array}{l}\text { 8. El nivel de esfuerzo } \\
\text { mental necesario para } \\
\text { evitar los errores en mi } \\
\text { trabajo es: }\end{array}$ & 4,00 & 3,71 & 3,24 & 3,92 & 4,40 & 2,617 & 0,046 \\
\hline \multirow{3}{*}{$\begin{array}{l}\text { Características } \\
\text { de la tarea. }\end{array}$} & $\begin{array}{l}\text { 6. El número de } \\
\text { interrupciones (llamadas } \\
\text { telefónicas, atender } \\
\text { público, otros compañeros } \\
\text { solicitando información, } \\
\text { etc.) durante la realización } \\
\text { de mi trabajo es: }\end{array}$ & 2,88 & 2,29 & 3,18 & 4,00 & 4,80 & 6,893 & 0,000 \\
\hline & $\begin{array}{l}\text { 7. La cantidad de } \\
\text { dificultades que se } \\
\text { producen cuando se } \\
\text { introducen nuevos } \\
\text { procedimientos de trabajo } \\
\text { o programas informáticos } \\
\text { es: }\end{array}$ & 3,75 & 3,29 & 3,41 & 4,08 & 4,00 & 2,487 & 0,055 \\
\hline & $\begin{array}{l}\text { 13. En mi trabajo, tengo } \\
\text { que hacer más de una } \\
\text { tarea a la vez: }\end{array}$ & 3,25 & 3,50 & 4,12 & 4,50 & 4,20 & 2,927 & 0,030 \\
\hline $\begin{array}{l}\text { Organización } \\
\text { temporal del } \\
\text { trabajo. }\end{array}$ & $\begin{array}{l}\text { 18. El tiempo del que } \\
\text { dispongo para tomar las } \\
\text { decisiones exigidas por mi } \\
\text { trabajo es: }\end{array}$ & 3,13 & 3,14 & 3,71 & 3,25 & 2,40 & 2,994 & 0,027 \\
\hline
\end{tabular}

*G1: Primaria, *G2: Secundaria, *G3: Preparatoria, *G4: Licenciatura, *G5: Posgrado

Tabla 14. Dimensiones / horas de trabajo

\begin{tabular}{|c|c|c|c|c|c|c|}
\hline \multirow{2}{*}{ Dimensión } & \multirow{2}{*}{ Reactivo } & \multicolumn{3}{|c|}{ Media } & \multirow{2}{*}{ F } & \multirow{2}{*}{$\mathrm{p}$} \\
\hline & & $\mathrm{G}^{*}{ }^{8}$ & $\mathrm{G} 2^{*}$ & G3* & & \\
\hline \multirow{2}{*}{$\begin{array}{l}\text { Demandas } \\
\text { cognitivas y } \\
\text { complejidad de la } \\
\text { tarea. }\end{array}$} & $\begin{array}{l}\text { 2. La cantidad de } \\
\text { memorización de } \\
\text { información y material que } \\
\text { requiere mi trabajo es: }\end{array}$ & 3,79 & 3,44 & 4,13 & 2,943 & 0,061 \\
\hline & $\begin{array}{l}\text { 3. El nivel de esfuerzo o } \\
\text { concentración mental que } \\
\text { requiere mi trabajo es: }\end{array}$ & 3,93 & 3,71 & 4,38 & 3,630 & 0,033 \\
\hline $\begin{array}{l}\text { Características de } \\
\text { la tarea. }\end{array}$ & $\begin{array}{l}\text { 10. Las tareas que realizo en } \\
\text { mi trabajo requieren una alta } \\
\text { concentración debido a la } \\
\text { cantidad de distracción o } \\
\text { ruido de fondo: }\end{array}$ & 4,00 & 3,24 & 4,00 & 4,302 & 0,019 \\
\hline
\end{tabular}

${ }^{*} \mathrm{G} 1: 8$ horas o menos, * ${ }^{\mathrm{G} 2}$ : Más de 8 hasta 10 horas, ${ }^{8} \mathrm{G} 3$ : Más de 10 horas

\section{Discusión}

En primera instancia, hay que señalar que la muestra no fue la que se estimaba, aunque no fue grande, arroja información interesante para analizar, y la cual se reforzó mediante la observación y entrevistas a los trabajadores en su ámbito laboral. Por otro lado, el valor de esta investigación radica en aspectos reales de la vida cotidiana del trabajador, ya que muchos instrumentos de medición son aplicados en situaciones controladas o fuera del 
contexto laboral y que limita al final en cierto grado el análisis posterior de la información.

En esta investigación se comprobó que la carga mental de trabajo tiene relación con el puesto de trabajo desempeñado, así como con las características demográficas y laborales en el contexto manufacturero automotriz. Esto tiene consecuencias en dos sentidos, en primera instancia en la persona, ya que su calidad de vida se verá afectada, y con ello sus relaciones sociales, también su desarrollo profesional estará en peligro por no contar con las habilidades físicas y psicologías para enfrentar con éxitos sus tareas en la empresa. En cuanto a la empresa, se verá afectada en la calidad de sus productos, ausencias de personal, imagen corporativa y rotación de personal.

En México este tipo de estudios no son aplicados por que no se conocen de fondo, solo en el ámbito académico se habla de tales conceptos sin que haya un impacto real en las empresas. El reto principal es en primera instancia acercarse a la industria y explicar los beneficios que aportaría un estudio de carga mental, sin que para ello haya grandes inversiones de recursos. La escala de medición se aplica en tan solo 15 minutos y se puede responder con lápiz en el mismo lugar de trabajo. Esto llevaría a romper la brecha entre la parte teórica y la práctica, por que obtendría información en contextos reales.

En general, los resultados que aporta esta investigación pueden utilizarse y compararse a otras realidades organizacionales o para abundar en el mismo. La gran ventaja del uso del instrumento ESCAM es que el análisis se puede realizar rápido e identificar las áreas a mejorar, ya sea mediante el rediseño de puestos de trabajo, capacitación del personal o reubicación del personal.

Ante todo, se tiene que proteger la integridad física y psicológica del trabajador, por el motivo más importante: porque es una persona. Ésta a su vez constituye lo más preciado de la comunidad en la que está inmersa dicha organización, lo que puede convertir a una empresa en una escuela en donde se aprenden y aplican valores

\section{References:}

Almirall, P. (2001). Ergonomía cognitiva. Apuntes para su aplicación en salud y trabajo. Caracas: Universidad Central Venezuela/INSTA.

AMIA, Asociación Mexicana de la Industria Automotriz, a.c. "Estadísticas a propósito de ... la industria automotriz". Recuperado de: http://www.amia.com.mx/

Bolger, N., De Longis, A., Kessler, R. C. \& Schilling, E. A., (1989). Effects of daily stress of negative mood. Journal of Personality and Social Psychology, 56, 808-818. 
Ceballos, P., Paravic, T., Burgos M. y Barriga, O. (2014). Validación de escala subjetiva de carga mental de trabajo en funcionarios/as universitarios. Ciencia y Enfermeria, 20(2), 73-82.

Charnock, D. \& Manenica, I. (1978). Spectral analysis of R-R intervals under different work conditions. Ergonomics, 21, 103-108.

Conway, T., Vickers, R., Ward, H. \& Rahe, R. (1981). Occupational stress and variation in cigarrete, coffee and alcohol consumption. Journal of Health a Social Behavior, 22, 155-165.

Cooper, C. \& Payne, R. (1980). Stress at work. Chichester: John Wiley and Sons.

Dombois, R. (1990). Economía política y relaciones industriales en la industria automotriz mexicana. In J. Carrillo (Coord.), La nueva era de la industria automotriz en México (pp. 35-63). México: El Colegio de la Frontera Norte.

Friedman M. \& Rosenman R. (1974). Type A behavior and your heart, Nueva York: Knopf.

Galster, S. (2004). An examination of complex human-machine system performance under multiple levels and stages of automation. Dissertation Abstracts International: Section B: The Sciences \& Engineering, 64(10-B), 2004, 5258. US: Univ Microfilms International.

González, E. (2006). Carga de trabajo mental y estrés en trabajadores de la industria electrónica. Tesis de doctorado. México: UNAM.

Gutiérrez, R. y Ángeles, Y. (2012). Estrés organizacional. México: Trillas.

Hanson, E., Schellekens, J., Veldman, J. \& Mulder, L. (1993). Psychomotor and cardiovascular consequences of mental effort and noise. Human Movement Science, 12, 607-626.

Hart, S. \& Wickens, C. (1990). Workload assessment and prediction. In H. R. Booher (ed.), MANPRINT: An emerging technology. Advanced concepts for integrating people, machines and organizations, 257-300. Nueva York: Van Nostrand Reinhold.

Hitchen, M., Brodie, D. A. \& Arnés, J. B. (1980). Cardiac responses to demanding mental load. Ergonomics, 23, 379-385.

Hurrell, J., Murphy, L., Sauter, S. \& Cooper, C. (1988). Occupational stress. Issues and developments in research. Philadelphia: Taylor \& Francis.

INEGI, Instituto nacional de estadística y geografía. Censos económicos 2014. Recuperado de: http://www.inegi.org.mx/

International Standard Organization. (1996). ISO 10075-2 Ergonomics principles related to mental workload. Part 2: Design principles. Suiza: ISO. Jamner, L., Shapiro, D., Goldstein, I. \& Hug, R. (1991). Ambulatory blood pressure and Heart rate in paramedics: Effects of cynical hostility and defensiveness. Psychosomatic Medicine, 53, 393-406. 
Jung, H. \& Jung H. (2001). Establishment of overall workload assessment technique for various tasks and workplaces. International Journal of Industrial Ergonomics, 28, 341-353.

Kirmeyer, S. \& Dougherty, T. (1988). Workload, tension and coping: moderating effects of supervisor support. Personal Psychology, 41, 125-129. Lauridsen, O. \& Tonnesen, T. (1990). Injuries related to aspects of shiftworking. A comparison of different off-shore shift arragements. Journal of Occupational Accidents, 12, 167-176.

Leino, P. \& Hanninen V. (1995). Psychosocial factors at work in relation to back and limb disorders. Scandinavian Journal of Work and Environmental Health, 21, 134-142.

Lundberg, U. (1995). Methods and applications of stress research. Technology Health Care, 3, 3-9.

Lundberg, U. \& Palm, K (1989). Workload and catecholamine excretion in parents of preschool children. Work and Stress, 3, 255-260.

Minowa H. (2000). The workload of computer system engineers and mental health. Sangyo Eiseigaku Zasshi, 42, 17-23.

Moray, N. (1982). Subjective mental workload. Human Factors, 24, 25-40. Neffa, J.C. (1988). ¿Qué son las condiciones y medio ambiente de trabajo? Propuesta de una nueva perspectiva. Buenos Aires: Hvmanitas-Ceil.

Pettigrew, T. (1988). Influencing policy with social psychology. Journal of Social Issues, 44, 205-219.

Repettí, R. (1993). The effect of workload and the social environment at work. In L. Goldberg, \& S. Breznit. Handbook of stress. Theorical and clinical aspects. New York: The Free Press.

Rocha, R. (2005). Carga mental laboral y psicotrastornos en trabajadores industriales Liberabit. 10(11), 83-89.

Rocha, R. (2006). Un punto de vista psicosocial sobre el trabajo en México. Liberabit, 12(12), 55-77.

Rodríguez, A y Roldán, J. (2014). Validez de un instrumento de carga mental mediante un piloteo en una muestra de trabajadores mexicanos. Tesis de licenciatura. México: UNAM.

Rollo, G., Díaz, D. y Fernández E. (2009). Desarrollo de una escala subjetiva de carga mental de trabajo (ESCAM). Revista de Psicología del Trabajo y de Las Organizaciones, 25(1), 29-37.

Rose, R., Jenkins, C. \& Hurst, M. (1978) Air traffic controller health change study. (FAA report no. AM-78-39) Washington D. C. Federal Aviation Administration.

Schabracq, M., Cooper, C., Travers, Ch. \& Van Maanen, D. (2001). Occupational health psychology: the challenge of workplace stress, United Kingdom: The British Psychological Society. 
Sluiter, J., Croon, E., Meijman, T. \& Frings-Dresen, M. (2003). Need for recovery from work related fatigue and its role in the development and prediction of subjective health complaints. Occupational and Environmental Medicine, 60, 62-70.

Téllez, A. (2001). Niveles de estrés y ansiedad en el personal médico, paramédico y de enfermería de la Cruz Roja Mexicana. Tesis de Licenciatura, Universidad Nacional Autónoma de México.

Theorell, T. \& Floderuss-Myrhed, B. (1977). Workload" and risk of myocardial infarction - a prospective psychosocial analysis. International Journal of Epidemiology 6, 17-21.

Tsang, P. \& Wilson, G. (1997). Mental workload. I: G. Salvendy (Ed.), Handbook of human factors and ergonomics. Wiley-Interscience.

Uribe, J. (2014). Clima y ambiente organizacional: Trabajo, salud y factores psicosociales. México: Manual moderno.

Vicencio, A. (2007). La industria automotriz en México. Antecedentes, situación actual y perspectivas. Contaduría y Administración, 221, 211-248. 